\title{
escrito porna.
}

\section{${ }^{1}$ Freeda Jusino-Sierra}

Bachillerato en Sociología, maestría en Trabajo Social y doctorado en Educación, todos de la Universidad de Puerto Rico. Labora como supervisora intermedia y evaluadora forense de alegaciones de abuso sexual infanto-juvenil, en el Programa de Apoyo a Víctimas de Abuso Sexual y sus Familias de la Universidad Carlos Albizu.

$\triangle$ freedajusino@gmail.com

(D) ORCID ID https://orcid.org/0000-0002-7483-9189

Cómo citar / citation: Jusino-Sierra, F. (2020). Educación sexual ¿tarea para los padres?: Abordaje desde una perspectiva crítica a la política educativa contemporánea de Puerto Rico. Voces desde el Trabajo Social, 8(1), 200-225. https://doi.org/10.31919/voces.v8i1.224

Recibido / received:

6 de septiembre de 2020
Revisado / reviewed:

3 de noviembre de 2020
Aceptado / accepted: 8 de diciembre de 2020

Derechos de autoría / Copyright: (c) 2020 Jusino-Sierra, F. Este es un artículo de acceso abierto y distribuido bajo los términos de la licencia y políticas de Creative Commons Attribution 4.0 International License 


\section{EDUCACIÓN SEXUAL iTAREA PARA LOS PADRES?: ABORDAJE DESDE UNA PERSPECTIVA CRÍTICA A LA POLIIIICA EDUCATIVA CONTEMPORÁNEA DE PUERTO RICO}

\section{Resumen}

$$
\text { G openaccess PeER-ReVIEWed }
$$

A través de este artículo, se abordará la educación sexual en las escuelas como parte de la propuesta expuesta en la Ley 85 del 2018: Ley de Reforma Educativa de Puerto Rico. Se expondrá un análisis reflexivo desde una mirada filosófica a través de los exponentes educativos clásicos. Se estará triangulando la educación sexual, con la política educativa y política social para visualizarlo a través del marco en la niñez. Esto será plasmado en un contexto cultural y se abordará en torno a la historia de la política pública de educación sexual, sexualidad y abuso sexual en Puerto Rico. En cuanto a los derechos de la niñez, se estará visualizando la educación sexual como parte inherente a estos fines. Todo lo anterior, será analizado desde un matiz crítico con una propuesta transformadora a manera de conclusión.

\section{Palabras claves}

educación sexual, ley 85 del 2018, política educativa, tabú, derecho de la niñez 


\section{Sumario}

Introducción. Ley 85 del 2018: Ley de Reforma Educativa de Puerto Rico. De la educación a la educación sexual: Una mirada desde la filosofía educativa. Sexualidad en el entorno social. Política educativa, política social y educación sexual: una mirada desde el marco en la niñez. Historia de la política pública de educación sexual, sexualidad y abuso sexual: Alcance en Puerto Rico. Necesidad de la educación sexual como derecho. Análisis crítico. Una propuesta transformadora a manera de conclusión. Referencias.

\section{IEN] SEX EDUCATION, ASSIGNMENT FOR PARENTS?: AN APPROACH FROM A CRITICAL PERSPECTIVE TO CONTEMPORARY EDUCATIONAL POLICY IN PUERTO RICO}

\section{Abstract}

Sexual education in schools will be addressed as part of the proposal set forth in Law 85 of 2018: Puerto Rico Education Reform Law through this article. A reflective analysis will be presented from a philosophical point of view through the classical educational exponents. Sex education will be triangulated, with educational policy and social policy to visualize it through the framework in childhood. This will be reflected in a cultural context and will be addressed around the history of public policy on sexual education, sexuality, and sexual abuse in Puerto Rico. Sexual education is being viewed as an inherent part of the children's rights. All of the above will be analyzed from a critical perspective with a transforming proposal as a conclusion.

\section{Keywords}

sex education, law 85 of 2018, educational policy, taboo, children's rights 


\section{Introducción}

Este artículo se aproxima, desde una perspectiva crítica, a la política educativa contemporánea en torno a cómo se expuso el tema de la educación sexual en la Ley 85 del 2018, Ley de la Reforma Educativa de Puerto Rico. El análisis de la serie ${ }^{9}$ 410 del Compendio de Políticas del Departamento de Educación sostendrá que ha existido una (des)vinculación con este estatuto. Se deja establecido que las Cartas Circulares fueron dejadas sin efecto para dar paso el Compendio a partir del 10 de agosto de $2017^{10}$. Se estará ejemplificando cómo políticas públicas aprobadas de educación sexual, sexualidad y abuso sexual no se han implementado (en su totalidad) como fueron propuestas. La ubicación teórica analítica integrará los aspectos socioculturales con los fundamentos de la educación. Esto, para problematizar el ideario de que los progenitores deben estar a cargo de seleccionar cómo educaran a sus hijos sobre la sexualidad. Para ello, se cuestionará cómo padres sesgados por los tabúes de la sexualidad que existen en las familias puertorriqueñas pueden impartir el tema

9 Listado de políticas públicas del Departamento de Educación. Se enumeran por "series" de acuerdo al tema o asunto.

10 La fecha está al entrar al enlace del Departamento de Educación de Puerto Rico https://www.de.pr. gov/?s=compendio+de+pol\%C3\%ADticas, no así en el enlace directo del Compendio como tal. de la educación sexual a sus hijos. Además, se presentarán propuestas para atender la necesidad de educación sexual de la población en las escuelas de Puerto Rico.

\section{Ley 85 del 2018: Ley de Reforma Educativa de Puerto Rico}

El 29 de marzo de 2018, dio inicio la Ley 85. En la misma, se expuso bajo el artículo 11.01, Derechos de los padres, tutores y encargados, que estos pueden "seleccionar, de forma exclusiva, la forma y manera que se educarán sus hijos respecto a la sexualidad y afectividad" (p. 79). Al revisar la Ley, no hay otra instancia en la que se vuelva a mencionar el tema de la educación sexual. No obstante, cuando se revisa el Compendio de Políticas del Departamento de Educación vemos que en la Serie 410, Currículo de Salud, su primer apartado pertenece al de Salud Sexual y su asunto explícito es "Política pública de Educación en Salud Sexual en las Escuelas Públicas del Departamento de Educación” (pp. 104-107). En su resumen, se especifica:

Se establece la política pública del DE respecto a la educación en salud sexual como parte del Programa de Salud Escolar, el cual como parte integral de su currículo está el desarrollar actividades educativas dirigidas a la educación sexual. Se propone el Modelo de Educación para la Salud Sexual Integral para la planificación e 
implementación del proceso de enseñanza-aprendizaje de forma científica y adecuada (Compendio de Políticas del Departamento de Educación, Serie 410, p. 104).

Ante lo leído, se problematiza lo siguiente: ¿Se vinculó la política pública educativa con las normativas de los currículos? ¿Hay una (des) vinculación directa? ¿A qué debe atenerse los/as directores/as de escuela, a la serie 410-a, o a la Ley 85 del 2018? A través de este artículo, se irán analizando los aspectos medulares del Currículo de Salud, Salud Sexual (Compendio de Políticas del Departamento de Educación, Serie 410, pp. 104-107).

\section{De la educación a la educación sexual: Una mirada desde la filosofía educativa}

Comencemos por la importancia de la educación en la sociedad. El educador clásico Dewey (1978) destacó que la educación es necesaria en la vida y que los seres vivos nos conservamos por renovación. Esto significa, que lo que antes era adecuado, no lo va a hacer necesariamente en el presente. En forma vinculante, Vygotsky (1997) destacó la necesidad explícita de tomar en cuenta el ambiente cultural, el bagaje cultural y la idiosincrasia de las personas al momento de impartir la educación. Se debe tomar en cuenta la época y la cultura en que se desarrolla el ser humano. De acuerdo a Gonçalves Salgado y De Souza (2018), se mantiene aún la "fuerza en los discursos sociales de protección, cuidado y educación de los niños" (p. 242), en la cual, se ve "la infancia como una 'etapa de la vida', como forma de afirmar 'verdades' sobre los sujetos que la viven y sobre cómo ‘deben' vivirla” (p. 242).

Teniendo como base lo ya presentado, es meritorio analizar lo que constituye la educación en la sociedad, lo cual no se podría desligar del pragmatismo (¿Cómo juzgamos esa verdad considerando sus efectos prácticos?). ¿Cuál sería el efecto "verdadero" de poner en práctica el que solo los padres eduquen sexualmente a sus hijos? Esto lo podríamos hilvanar a lo que es el pragmatismo. Una de las caracterizaciones del filósofo Richard Rorty (1982) en torno al pragmatismo fue la aplicación del antiesencialismo a nociones como: verdad, conocimiento, lenguaje, moralidad, y semejanzas de objetos de especulación filosófica. Ante esto, nos podemos plantear, ¿qué era lo que realmente se deseaba implementar con la Ley 85 del 2018 ? ¿Entenderían los progenitores qué era lo que el Gobierno deseaba para los niños y su derecho a recibir educación sobre la sexualidad? ¿Sería útil la educación sexual casera para propósitos de una política 
pública salubrista lo suficiente como para evitar embarazos, contracción de enfermedades/infecciones de transmisión sexual, entre otros?

Aún sin tener respuestas concretas para estas preguntas, retomemos a Rorty (1982) y otra de las categorizaciones del pragmatismo. Él indicaba que no había diferencia epistemológica entre la verdad de lo que es y la verdad de lo que debe ser, como tampoco había diferencia metafísica entre hechos y valores, ni diferencia metodológica entre la moralidad y la ciencia. Ante esto, es válido plantearse: ¿va a tono enseñarle educación sexual casera a los niños con los derechos humanos? ¿Cuál sería esa "verdad" de cara al pragmatismo y la Ley 85? De acuerdo a Rorty, la tradición epistemológica cometía un error cuando buscaba la esencia de la ciencia e intentaba reducir la racionalidad a una regla. Y si es así, entonces, ¿quién debe determinar cómo o quiénes están preparados para impartir educación sexual (educadores adiestrados o padres)?

Como se ha expuesto, hay una (des)vinculación con las normativas (series) del Departamento de Educación y la política pública educativa. Ni en la ley 85 del 2018, ni en el Compendio de Políticas del Departamento de Educación se desplegó una definición de educación sexual. Habiendo expuesto esto, se reconoce que la educación sexual es un enfoque culturalmente relevante $\mathrm{y}$ apropiado a la edad del participante, que enseña sobre el sexo y las relaciones interpersonales a través del uso de información científicamente rigurosa, realista y sin prejuicios de valor. La educación en sexualidad brinda a la persona la oportunidad de explorar sus propios valores y actitudes y desarrollar habilidades de comunicación, toma de decisiones y reducción de riesgos respecto de muchos aspectos de la sexualidad (UNESCO ${ }^{11}, 2014$, p. 38).

La política educativa pudiera ser hilvanada a todo lo anterior. El medio ambiente y la sociedad en que se desarrollan los niños hoy son diferente a la que se desarrollaron sus padres o tutores. En un estudio publicado por Montero-Ossandón et al. (2017), se halló que el conocimiento sexual de los jóvenes era uno amplio por la exposición de acceder a la información rápidamente no solo a través de su medioambiente sino también por las redes sociales. En otro estudio hecho en Puerto Rico sobre el tema de sexualidad y religión, la autora destacó en su revisión literaria, la presencia de la glorificación del sexo en los medios de comunicación y que "los padres y madres muestran incapacidad para

11 Organización de las Naciones Unidas para la Educación, la Ciencia y la Cultura. 
comunicarse efectivamente con sus hijos e hijas" (Morales, 2018, p. 21). Por lo cual, tal pareciera que los padres pudieran estar en desventaja antes sus hijos en torno a lo que sus vástagos conocen en torno a la sexualidad.

\section{Sexualidad en el entorno social}

La sexualidad tiene que ver con los valores, creencias y actitudes. Por lo cual, lo que es el tabú, la socialización, la influencia de la religión y los cambios generacionales, merecen el análisis al amparo de lo que pretende la Ley 85 del 2018 con delegarle a los padres la educación sexual de sus hijos. Además, se visibiliza que los niños están expuestos a la "presión de pares y la reproducción de las conductas que se modelan en la televisión, el cine y las revistas locales e internacionales" (Morales, 2018, p. 36).

En la disertación de Nieves (2012), se halló que al comparar tres políticas de educación sexual, había tabú en torno a la sexualidad y el discurso. Por otro lado, en la disertación de De Jesús (2018), se reseñó la preocupación del "tabú social en [que] permea a la niñez/ juventud en temas vinculados a la sexualidad, lo que a su vez acrecienta su silenciamiento en asuntos como el abuso sexual, ello a pesar de que estos, la niñez y juventud, hayan sido los victimizados" (p. 129).
En la cultura puertorriqueña, hay tabú sobre la sexualidad (Fontes \& Plummer, 2010). No hablarles a los niños sobre la sexualidad podría esconder situaciones de abuso sexual intrafamiliar, pudiera acarrear embarazos en los adolescentes, y pudiera incrementar la exposición a enfermedades/infecciones de transmisión sexual. A esto, se le añade que el comportamiento sexual está influenciado por factores individuales, familiares y ambientales (Miragoli et al., 2017). Si no se toma en cuenta el mejor bienestar de los niños, al ser creada una política educativa como esta, pone de manifiesto que no se les reconoce como sujetos de derecho. Tal pareciera que están bajo el amparo del adultismo ${ }^{12}$, ya que se les reconoce como objetos del derecho, y no como ciudadanos sujetos de derecho, de su propio derecho (De Jesús-Rosa \& JusinoSierra, 2017). Y como planteara Freire (1997), "enseñar exige respeto a la autonomía del ser del educando [...] el respeto a la autonomía y a la dignidad de cada uno es un imperativo ético y no un favor" (p. 58).

En un estudio realizado en Estados Unidos en el que se comparó los conocimientos de los niños sobre la terminología genital, encontraron que ninguno de los niños de habla

12 El adultismo es la dinámica en la cual el mundo de la adultez oprime al mundo de la niñez y juventud (De Jesús, 2018). 
hispana conocía los términos correctos para los senos, el pene o la vulva, sugiriendo un vacío en la educación sexual en los hogares de habla hispana (Kenny \& Wurtele, 2008; en Kenny \& Wurtele, 2013, p. 450 ). Otros investigadores han descrito que el silencio sexual ocurre a menudo en las familias hispanas (Vanoss Marín, 2003; en Kenny \& Wurtele, 2013, p. 45013) y parece ser un tabú contra la discusión de la sexualidad en muchas culturas latinas, y la comunicación de los padres con respecto a la sexualidad (Kenny \& McEachern, 2000; Russell, Lee, \& el Grupo de Trabajo para la Prevención del Embarazo en los Jóvenes Latinos, 2004; en Kenny \& Wurtele, 2013; p. 450). Fontes y Plummer (2010, p. 497) señalan que la educación sexual en familias puertorriqueñas ha sido descrita como "¡Chitón!, ¡De eso no se habla!” ("Hush! We don't speak about that!"). Se desconoce hasta qué punto esta tradición cultural de relativa incomodidad con las discusiones sexuales y las relaciones sexuales puedan impactar las conductas sexuales en el desarrollo de la sexualidad de los niños, según se expuso en ese artículo.

Se deja establecido que en Puerto Rico, el abuso sexual infanto-

13 Se le ha colocado los números de página a los parafraseos según lo recomendado en la reunión del 29 de marzo de 2019. juvenil ha sido trabajado en forma revictimizante (López \& Rivero, 2015). El abuso sexual intrafamiliar es uno de los más frecuentes y con este se acarrea el tabú de la sexualidad. Tener (2018) señaló que las reacciones familiares o sociales negativas no pueden abordarse de forma aislada, sino que deben considerarse en el contexto de la tendencia social a evitar y colocar tabúes en los comportamientos, actitudes y formas de pensar que amenazan el orden social (p. 3). De acuerdo a Eisikovits et al. (2017), la literatura empírica sobre la relación diaria entre las mujeres adultas sobrevivientes del abuso sexual intrafamiliar 14 y sus agresores sugiere que el tabú es uno real (p. 217).

En forma vinculante, no se puede desligar la sexualidad de la socialización de género y masculinidad. Rondina (2017) señala que como parte de esa socialización también es enseñado el "ser macho es ser fuerte, saber beber, ser mujeriego, tener hijos -prueba incontestable de su virilidad- y ser proveedor de la casa" (p. 88). Tal como indicara ella, la socialización se considera para que concretice los derechos en las políticas sociales. La educación es un derecho, y tal como fue expuesto en la misma la

$14 \mathrm{Al}$ exponer abuso sexual intrafamiliar, se trata que los niños y jóvenes sean abusados por familiares que usualmente viven con ellos. 
Ley 85 del 2018 (p. 1), "[los marcos que] fundamentará[n] el sistema de enseñanza en Puerto Rico [...] atenderá las necesidades actuales y futuras de nuestra sociedad; reformular[á] el sistema educativo en función del estudiante como centro y eje principal de la educación", sería útil bajo esta premisa hacer valer lo que se expone en la Carta de los Derechos del Niño (Ley 338 [L.P.R. 15], 1998). Este, en su ser individual, pero como parte de la sociedad,

debe contar con el apoyo y respaldo de la comunidad y el Estado. Este compromiso de la familia, la comunidad y el Estado resultará en la formación de un niño sano, más balanceado emocionalmente, y mejor preparado para constituirse, no solo en un ciudadano productivo, sino en uno activamente comprometido con el mejoramiento personal y colectivo (párr. 4).

En torno a las influencias de la religión, en un estudio publicado por Machado das Neves y Ribeiro da Silva (2018), se encontró que los maestros abordaron el tema de los prejuicios para la atención de proyectos de igualdad de derechos para población LGBT en la escuela. Destacaron que los conservadores religiosos, en asociación con parlamentarios fundamentalistas en países europeos y latinoamericanos, buscan aprobar leyes que prohibieran la posibilidad de la implantación de las políticas de género y sexualidad en el contexto escolar, bajo un argumento peyorativo y difamatorio de que esas políticas apuntan a destruir familia tradicional y acabar con la inocencia de los niños (Machado das Neves \& Ribeiro da Silva, 2018, p. 10). Lo hallado en este estudio fue la necesidad de la implementación de políticas públicas educativas para asegurar derechos y minimizar prejuicios (p. 10).

¿Qué pasaría con los padres que practican el fundamentalismo religioso (interpretación literal de los textos religiosos)? MonteroOssandón et al. (2017) expusieron que en "el ámbito de la educación sexual, la doctrina católica establece que esta debiera realizarse bajo la supervisión de los padres, tanto en la casa como en los centros educativos escogidos por ellos" ( $p$. 350). Informaron que, en este sentido, la contribución de los educadores será preparar los jóvenes a "vivir la castidad", "respetar y promover el 'significado esponsal' del cuerpo" (p. 350). Por lo cual, "la Iglesia se opone a un sistema de información sexual separado de los principios morales" (Montero-Ossandón et al., 2017, p. 350).

El otro aspecto fundamental sería la toma en consideración de los cambios generacionales. Nieves (2010)

15 Leyes de Puerto Rico. 
publicó un estudio sobre la política pública, la educación sexual y los adolescentes en Puerto Rico. Como parte de las conclusiones, sostuvo:

Los métodos de educación sexual deberían responder, entre otros aspectos, al desarrollo fisiológico del adolescente y al momento histórico que se está viviendo, ya que esto depende, en gran medida, de la sociedad y de las tensiones particulares, políticas y económicas de la época. [...] El contenido curricular sobre educación en salud sexual no puede permanecer estático, sino que tiene que actualizarse y adecuarse según las características generacionales. La política establece que se debe recopilar estudios para identificar el comportamiento sexual de riesgo y sugiere un ejemplo de niveles de riesgo. [...] La educación sexual no puede limitarse a la educación sobre aspectos fisiológicos, sino que debe referirse a todos los elementos que remiten a la sexualidad. [...] las implicaciones de las construcciones sobre el género, [...] deberían ser materia curricular (p. 152).

Se observa lo expuesto por el filósofo educativo Dewey (1978) respecto a que las leyes y normativas deben tener su base en los cambios sociales y antropológicos.

La continuidad de toda experiencia, mediante la renovación del grupo social, es uno literal. La educación en su sentido más amplio, es el medio de esta continuidad de la vida. Cada uno de los elementos constitutivos de un grupo social [...] nace inmaduro, indefenso, sin lenguajes, creencias, ideas ni normas sociales. Cada individuo, cada unidad de portadores de la experiencia vital de su grupo desaparece con el tiempo. $Y$ sin embargo la vida del grupo continúa (Dewey, 1978, p. 10).

\section{Política educativa, política social y educación sexual: una mirada desde el marco en la niñez}

Para la creación de una política pública educativa, se debe identificar las causas del problema para generar alternativas para solución (Pulido-Chavez, 2017). Además, "las finalidades de una política pública buscan dar respuesta a las demandas de la acción pública, a las necesidades, generando acciones de impacto sobre el terreno para fortalecer lo público, lo que a su vez nutre el quehacer de los trabajadores sociales" (Cruz-García \& LópezOpina, 2019, p. 89). Se desconoce si en la creación de la Ley 85 del 2018 , se tomó en cuenta el número de embarazos en adolescentes y cómo impactaría el que no existiera una obligación en las escuelas de enseñar sobre la educación sexual. Además, si fue tomado en consideración los aspectos sociales del tabú sobre la sexualidad, esto pese a que en el currículo de Salud Sexual sí se explicitó en el inciso 3(b): "facilitar que 
el estudiante comprenda plenamente el sentido de responsabilidad que implica tener relaciones sexuales y demostrar disposición para asumir comportamientos saludables, basados en factores protectores" (Compendio de Políticas del Departamento de Educación, Serie 410, p. 105).

La cultura no se mantiene; es una cambiante. Indicaba Dewey (1978) que los hombres viven en una comunidad por virtud de las cosas que tienen en común; y la comunicación es el modo en que llegan a poseer cosas en común. Lo que han de poseer en común con el fin de formar una comunidad o sociedad son objetivos, creencias, aspiraciones, conocimientos -una indulgencia común- una semejanza mental como dicen los sociólogos. Tales cosas no pueden pasarse físicamente de unos a otros [...] no pueden compartirse [...] [físicamente] la comunicación que asegura la participación en una inteligencia común es la que asegura disposiciones emocionales e intelectuales semejantes, como modos de responder a las expectaciones y a las exigencias (p. 12).

La brecha es la incongruencia entre la Política Pública Educativa y el Compendio de Políticas del Departamento de Educación. Se destaca que en el currículo de Salud Sexual, se expuso: "El modelo integral y formativo de la educación en salud sexual requiere incluir los hallazgos de la sicología al proceso educativo para crear programas que respondan a la realidad de la niñez y adolescencia de nuestra época" (Compendio de Políticas del Departamento de Educación, Serie 410, p. 104). El tener conocimiento es una ventaja para el desarrollo saludable de los estudiantes del sistema de educación pública. En la Carta de los Derechos del Niño (Ley 338 del 1998 [L.P.R.]) se establece que se "aspira a que en el futuro, nuestro pueblo sea más sano, más equilibrado y más feliz" (párr. 1). Smith (2000) indicó que cuando se vinculan los derechos de los niños y la política pública, surge el debate público lo cuál visibiliza las necesidades de esta población y se puede observar un compromiso emergente para mejorar la calidad de sus vidas ( $p$. 316). Sostuvo que cuando se hacen políticas públicas que involucran a la niñez, estas se ven trastocadas por tensiones y perspectivas conflictivas, ya que el poder y su uso deben ser para la comprensión de la niñez, con un enfoque de la política infantil, y centrado en la educación, y esto pocas veces se da (p. 317). Además, señaló que la preocupación por los niños y su bienestar ha avanzado en la agenda política (pp. 317, 323). Por lo cual, la posibilidad de considerar la infancia como un área de debate en las políticas públicas debe ser viable 
(p. 316). Postuló que los objetivos y las aspiraciones políticas no deben socavar las necesidades e intereses primarios de los niños (p. 318). Añadió que, de manera desvinculada, los niños no toman parte en la creación; el poder es de los adultos al igual la influencia (pp. 318-319, 321-322). Pero, en el Currículo de Salud sí se tomó en cuenta el derecho de "recibir una información correcta sobre sexualidad" (Compendio de Políticas del Departamento de Educación, Serie 410, p. 104).

En la política educativa, se deben tener presentes las normas legales y profesionales, y la teoría de la justicia social, entre otros (Gardiner \& Tenuto, 2016 , p. 7). Se debe asegurar, bajo una cultura educativa racional, que los niños cuenten con esos derechos humanos que tanto se mencionan al ventilar cualquier asunto en una organización educativa. "A los niños se les hace difícil y algunas veces imposible, hacer valer esos derechos" (Ley 338, 1998 [L.P.R.], párr. 2). Para poder ubicar esto, sería conveniente mirarlo desde la perspectiva funcionalista. Es a la luz de esta que se concibe a la sociedad como "un sistema u organismo ordenado e integrado compuesto por partes interdependientes (subsistemas, instituciones, roles sociales) que contribuyen a través de sus funciones especializadas $y$ complementarias a la integración, cohesión, estabilidad, productividad, mantenimiento y desarrollo de la sociedad como un todo" (Torres, 2012, párr. 10). Por lo cual, podría ser preocupante el que los padres "no quieran educar" a sus hijos a nivel sexual.

A raíz de lo ya presentado, ¿se soslayaría lo ya exhibido en el currículo de Salud Sexual en cuanto a "facilitar el desarrollo de destrezas que permiten prevenir la violencia y el abuso sexual" (Compendio de Políticas del Departamento de Educación, Serie 410, p. 105)? La educación sexual se reconoce como un campo de pericia, y aunque fue contemplado en el currículo en Salud Sexual, no lo fue en la Ley 85 del 2018. Se presume que el personal docente debe ser capacitado en "el tema de la educación sexual [y] la sexualidad responsable [...] [y se incluya] en los planes de trabajo de todos los niveles" (Compendio de Políticas del Departamento de Educación, Serie 410, p. 106).

¿Y los derechos de los niños? Ver a los niños como sujetos de su propio derecho y no como objetos del derecho del Estado es importante (De Jesús-Rosa \& Jusino-Sierra, 2017, pp. 522, 528-529). Si tomamos en cuenta lo anterior, estamos viendo cómo una política pública educativa, aparentemente, redactada sin tomar en cuenta el niño con sus 
derechos, puede invisibilizarlo como portador de esos derechos, derecho a conocer, derecho a ser socializado en forma humanizada, adecuada y políticamente correcta a nivel educativo. Los padres, en general, tienen sesgos y prejuicios en torno a la sexualidad. Esto no es un tema oculto. Rondina (2017) indicó que los seres humanos somos depositarios de nuestra memoria familiar, lo que vivimos, lo que escuchamos y lo que nos ha sido trasmitido (p. 360). La identidad se forma a través de esa memoria. Si los padres no han recibido educación formal sobre la sexualidad, ¿qué es lo van a transmitir a sus hijos? ¿Cómo es lo que van a hacer?

\section{Historia de la política pública de educación sexual, sexualidad y abuso sexual: Alcance en Puerto Rico}

En Puerto Rico, ya se había tomado en cuentala educación sexual como parte de las responsabilidades del Estado hacia la niñez. MartínezRamos (2013) esboza la historia de las políticas educativas relacionadas con género en Puerto Rico. Según expuso, tanto ella como otras educadoras, tuvieron varios traspiés para hacer valer la ley y en un momento dado, y fueron objetos de crítica, pues se pensaba que querían desmantelar la familia tradicional ( $p$. 21). Se dieron talleres en las escuelas en torno al tema de la sexualidad y el género, pero tuvieron muchas dificultades en que se pudiera comprender de qué se trataba, pues hubo mucha presión hacia la política pública relacionada con género, de parte de los grupos religiosos. También, se resalta la investigación llevada a cabo por Nieves (2010), sobre la política pública de educación en salud sexual en Puerto Rico, su implementación, proceso educativo y visión sobre los géneros. Este halló dificultad en que se hicieran valer la política pública sobre educación sexual. Se encontró que la misma no estaba siendo implementada ( $p$. 150). Hallazgo similar se encontró en la investigación de Ortiz (2016) en cuanto a algunos aspectos que pudieron ser implementados y otros no (pp. 198-199) en torno a la política pública de la educación sexual en varias escuelas.

Al destacar varias particularidades de la historia y la política educativa enmarcada en sexualidad, podemos señalar que en la carta circular núm. 3-2004-2005, Política Pública de Educación en Salud Sexual en las Escuelas Públicas del Departamento de Educación, se había expuesto que "todo niño y niña ha de tener acceso equitativo a la información, los servicios sociales y la educación para promover su desarrollo personal y su salud sexual integral" (p. 8). En el 2011, en la Cámara de Representantes se presentó la 
Resolución 1629, para ordenar a la Comisión de Asuntos de la Juventud de la Cámara de Representantes de Puerto Rico, llevar a cabo un estudio sobre la implantación de las disposiciones contenidas en esta carta circular, a fin de constatar la administración y funcionamiento de los planes estratégicos a corto y largo plazo para el ofrecimiento de programas de educación sexual desde el nivel elemental hasta el superior. En la búsqueda realizada, no se halló cómo concluyó. Posterior, la Carta Circular 3-2004-2005 fue derogada y se estableció la Carta Circular Núm. 2-2011-2012, Política pública de educación en salud sexual en las escuelas públicas del Departamento de Educación. En la misma se exponía el Modelo de educación para la salud integral (pp. 6-9).

Luego, se estableció la carta circular 19-2014-2015, la cual trataba sobre la política pública en torno la equidad de género y su integración al currículo del Departamento de Educación de Puerto Rico como instrumento para promover la dignidad del ser humano y la igualdad de todos y todas ante la ley. La misma fue creada con una amplia base legal e incluía la Ley 75, del 2007, Ley para ofrecer talleres educativos en todos los niveles del Sistema dirigidos a prevenir el abuso sexual infantil. No obstante, en el 2016, esta normativa fue "dejada sin efecto" a través de la Carta Circular 32-2016-2017 (p. 2), Política pública sobre la equidad de género y su integración al currículo del Departamento de Educación de Puerto Rico como instrumento para promover la dignidad del ser humano y la igualdad de todos y todas ante la ley. Seguía en pie la normativa de la Carta Circular 2-2011-2012.

En tres disertaciones doctorales revisadas (De Jesús, 2018; Nieves, 2012; Ortiz, 2016), también se puso de manifiesto que aunque existía la política pública de sexualidad, la misma no necesariamente se ponía en vigor. En la disertación de Nieves (2012), se halló que en la escuela solo se hablaba de cómo evitar embarazos y el uso de profilácticos al momento de hablar de sexualidad (pp. 94, 101, 123). En la disertación de Ortiz (2016), se expuso que los estudiantes sí estuvieron de acuerdo en que se les hablaba de métodos anticonceptivos, pero no les decían cómo usarlos, por lo cual carecían del conocimiento (p. 210).

Nieves (2012) destacó que, en el hogar, si existía mucha confianza con las madres, estas podían hablar de sexualidad pero era basado en lo mismo, en evitar la paternidad a temprana edad (pp. 87-88). Él halló, además, que aunque para ese momento existía una política pública sobre educación sexual, la 
misma no se estaba implementando tal como había sido propuesta (pp. $131,133)$; ya que había problemas en implementar políticas públicas (p. 162). Hallazgo similar en torno a otra política para trabajar con el abuso sexual infantojuvenil en la Isla fue expuesto por De Jesús (2018). En esta disertación, además de que se estaba trabajando en forma desarticulada la atención del abuso sexual infanto-juvenil, el tabú de la sexualidad fue un tema emergente. De Jesús (2018), también, halló dificultades en la implementación de la ley estudiada por él (pp. 219, 222223, 226).

\section{Necesidad de la educación sexual como derecho}

Como ya se mencionara, además de los juiciosy prejuicios delos padres, la educación sexual debe ser provista por personas capacitadas para eso. El dejar ignorantes a los estudiantes en torno a este tema, sería dejarlos desprovistos de sus derechos. Según indicaron Steinharta et al. (2013), los programas de educación sexual de buena calidad pueden desempeñar un papel importante en la reducción de los riesgos subyacentes y las vulnerabilidades que afectan el embarazo no deseado; la transmisión de infecciones de transmisión sexual y las relaciones sexuales coercitivas o abusivas (p. 630). Una educación sexual comprehensiva a los jóvenes puede desarrollar en ellos la capacidad de tomar decisiones informadas sobre sus vidas sexuales. Estos autores destacaron que los programas de educación sexual facilitan la distribución de información precisa, permitiendo la toma de decisiones conducentes al ejercicio saludable y placentero de la sexualidad. En esta misma línea de atribución, postulan que la escuela, como lugar importante de socialización, le puede dar a los estudiantes la oportunidad de cuestionar comportamientos o estereotipos que restringen su autonomía y diversidad, en muchos casos amenazando su salud sexual y reproductiva (Steinharta et al., 2013, p. 631). Señalan que, debido a que los niños deben asistir a las escuelas, este es el escenario ideal para la educación sexual. Aun así, reconocen que pese a que es lo más recomendado y avalado por la UNESCO, en muchas escuelas no se imparte educación sexual. En el Currículo de Salud, se avala la contemplación del "desarrollo de destrezas socioemocionales que propicien una sexualidad saludable" (Compendio de Políticas del Departamento de Educación, Serie 410, p. 104), pero no se observa cómo fue tomado en cuenta esto en la Ley 85. Por lo cual, se entiende las responsabilidad de la educación sexual debe ser una de 
responsabilidad compartida, pero la base debe ser dirigida por la escuela, con los educadores adiestrados en el tema.

Se reviste de importancia lo anterior ya que es en la escuela "donde el menor se desarrolla y pasa la mayor parte del tiempo" (López \& Rivero, 2015, p. 205). Y sí, es cierto que la escuela es solo "un medio $y$, comparado con otros factores, un medio relativamente superficial" (Dewey, 1978, p. 12) para poder dejarle solo a la escuela la educación sexual de los niños. Pero, de igual forma, podría parecer superficial dejarle la educación sexual solo a los padres. $Y$ es que en un estudio publicado en el 2010 por Wilson y colaboradores, se halló como un gran problema el que esto se pudiera llevar a cabo (p. 59). Los participantes del estudio (padres y madres) identificaron que se sentían incómodos al hablar sobre el sexo con sus hijos, pensaban que otra persona podría hacerlo mejor y tenían miedo que con el hablar sobre el particular ellos animarían a sus hijos a tener relaciones sexuales (p. 59). Otras barreras identificadas con una codificación mínima fueron: (a) que los padres no sabían lo suficiente para hablar del tema, (b) que tenían poca comunicación con sus hijos en general, (c) que estaban demasiado ocupados, y (d) que consideraban a sus hijos demasiado pequeños para hablar en torno a la sexualidad (p. 60). Según lo expuesto por estos autores, las principales barreras que los padres mencionaron fueron (a) la percepción de que sus hijos no estaban listos para hablar sobre el sexo y (b) la incertidumbre sobre cómo hablar de la sexualidad (pp. 59-60). A lo anterior, se le añade los hallazgos de la disertación de Ortiz (2016, p. 215), en torno a las siguientes barreras: (a) puede que haya una normalización entre las diferencias de los hombres y las mujeres, lo cual cimienta la desigualdad y el rol tradicional de los géneros, (b) algunos/as padres/ madres no saben definir lo que es salud sexual, y (c) la creencia que la salud sexual es solo el acto sexual, entre otros.

En otro estudio hecho en España en torno a la sexualidad en los jóvenes y lo que se habla en el hogar, Venegas (2013) halló que, según los datos recopilados, los jóvenes enfrentan los cambios de desarrollo en gran parte a través del silencio y la inhibición (p. 576). Ella destacó que el discurso familiar sobre la sexualidad identificado por los jóvenes hace hincapié en la evitación, la prevención y el "cuidado", habiendo poca referencia de la sexualidad (p. 576). Según indicó, la mayoría de los participantes informaron que sus familias definían negativamente la sexualidad o la negaban por completo (p. 576). 
Señaló que los jóvenes criados en una atmósfera como esta podían sentirse intrigados por el sexo, pero también tenían menos experiencia, y más estereotipos y conceptos erróneos sobre la sexualidad ( $p$. 576). Según los datos expuestos en los resultados, parece haber dos factores principales que influyen en la discusión de la sexualidad en el hogar con un adulto. Estos son: (a) la identificación de género porque las familias están más dispuestos a aceptar la participación de los niños en el sexo que las niñas y (b) la identificación intergeneracional porque los adultos ya han experimentado las situaciones que los jóvenes están teniendo (p. 576). Se destacó la culpa hacia las jóvenes sobre el embarazo (y no hacia los varones) (p. 577). Y se enfatizó los prejuicios hacia la homosexualidad si en el hogar no aceptan esta orientación sexual (p. 577).

Otro estudio realizado en Gran Bretaña, tuvo como base el embarazo en adolescentes y la educación sexual. El foco era la promoción de sensibilización para estimular el debate sobre el currículo, el cambio y el desarrollo de políticas (Littleton, 2014, p. 181). Esta autora destacó que la educación sexual debe entenderse desde una perspectiva amplia del mantenimiento de la salud reproductiva a lo largo de la vida de una persona, por lo cual el conocimiento apropiado debe darse desde temprano con un plan educativo (p. 185). Se identificó que esta meta curricular era tan importante como cualquier otra (p. 185).

\section{Análisis crítico}

A la luz de lo antes presentado, la crítica a la Ley 85 del 2018 es la desvinculación directa entre lo que está escrito en ella (los padres "pueden seleccionar, de forma exclusiva, la forma y manera que se educarán sus hijos respecto a la sexualidad y afectividad", p. 79) y lo que se halla en el Compendio de Políticas del Departamento de Educación. Se desconoce si la ley fue escrita tomando en cuenta el contexto puertorriqueño en torno al aspecto de la sexualidad y el tabú. Las políticas públicas educativas no deben descansar en redacciones de corte "moral" sin tomar en cuenta la cultura y la historicidad de donde serán implementadas. Al ir analizando lo ya esbozado, se percibe que hubo una invisibilización de la sociología y psicología educativa en esta partícula de la Ley.

Es preocupante pues se desconoce si realmente se tomó en cuenta lo sociocultural. Se supone que se haya considerado los acontecimientos diarios, lo cual es relevante al momento de crear, implementar y evaluar una política pública. Siendo la educación la base 
de la socialización, ¿es adecuado dejarles a los padres la educación sexual de sus hijos? En el estudio realizado por Mazuera-Arias (2018) se halló que la educación sexual "impartida por los padres es limitada, se restringe a los aspectos biológicos y de autocuidado"; además de que "las adolescentes que manifestaron tener mala y muy mala relación con la familia de origen NO recibieron educación sexual en la familia" ( $p$. 182). Se reconoce que la educación formal no puede ser invisibilizada en estos momentos históricos de Puerto Rico. En la Ley para la Seguridad, Bienestar y Protección de Menores (246 del 2011 [L.P.R.]), se expuso en el artículo 2 (Política Pública) que los niños, las niñas y los adolescentes

tienen derecho a [...] una buena calidad de vida y a un ambiente sano, en condiciones de dignidad y goce de todos sus derechos en forma prevalente. La calidad de vida es esencial para su desarrollo integral, acorde con la dignidad de ser humano. [...] Este derecho supone la generación de condiciones que les aseguren [...] la educación [...]. El Estado desarrollará política pública orientada hacia el fortalecimiento de los menores, proveyendo para que se establezcan esfuerzos razonables de apoyo y fortalecimiento a las familias en la prevención de la violencia y en la promoción de los valores que permiten una convivencia fundamentada en el respeto a la dignidad humana y al valor de la paz.

Hay una desvinculación de la Ley 85, con la sociología y antropología educativa. Este tipo de desarticulación va en detrimento de los derechos humanos de los niños, lo cual lleva hacia el adultismo explícito. La educación sexual en manos exclusivas de los padres, podría ser un error, y, quizás, la niñez ante el fundamentalismo religioso, el abuso sexual y el desconocimiento sobre la sexualidad estaría en una posición más vulnerable de la que actualmente ocupa.

\section{Una propuesta transformadora a manera de conclusión}

Al dar una mirada reflexiva a nivel educativo y sociológico nos encontramos conqueel quelos padres tengan la exclusividad de seleccionar cómo se van a educar a sus hijos podría traer efectos negativos a la salud, no solo sexual, sino emocional a los niños. Todo lo relacionado a educación sexual debe ser adaptado a la realidad contemporánea, además de "conocer la realidad que cada generación vive o ha vivido, y recoger y reciclar los aprendizajes que se pueden obtener" (UNESCO, 2014, p. 9). Por lo cual, es meritorio considerar las implicaciones del tabú, la socialización, la influencia de la religión y los cambios generacionales; 
y esto no debe invalidar el que los padres tengan participación en la misma.

Por lo antes expuesto, la propuesta para atender la necesidad de educación sexual en las escuelas debe tener su base a nivel transversal. Se propone que el tema de la sexualidad sea uno inclusivo en todas las materias; se debe tocar el tema en cada categoría que se trabaje con la sexualidad. Para esto, se necesita crear un currículo riguroso, abarcador y coherente, y que sea lo suficientemente flexible como para poder aplicarlo a cada materia; por lo cual debe ser comprehensivo. A nivel de transversalidad, un ejemplo sería que en la clase de Matemáticas, cuando se vayan a realizar ejercicios de cálculos, se pueda hacer el cálculo del periodo menstrual. Otro alcance sería, que en la clase de Ciencias se trabaje con las enfermedades/ infecciones de transmisión sexual. En lo que cubre las ciencias sociales como lo son los Estudios Sociales y la clase de Historia, se trabajen aspectos de la sexualidad y la (in)equidad del género. Se reconoce lo hallado en la disertación de Ortiz (2016) en torno al posible desconocimiento que tienen los maestros al impartir el tema de la sexualidad ("los/as maestros/as de materias diferentes a las de salud no tienen la capacitación suficiente en los temas de sexualidad. Esto es avalado por varios/as de los/as directores/as escolares, maestros/ as y madres participantes", p. 203). Por lo cual, el adiestramiento a los educadores resultaría indispensable.

Reconociendo que las políticas públicas son creadas a base de problemas reales y que se toma en cuenta cada aspecto de la historia y la cultura, la historicidad educativa en Puerto Rico en torno a temas de sexualidad debe ser viable. Se debe tomar en consideración todas las vicisitudes que hubo en un pasado al intentar adiestrar a los educadores (Martínez-Ramos, 2013, pp. 20-21) y tener presente lo difícil que puede ser no solo la creación, sino también la implementación y la evaluación de leyes con base en los derechos de los niños y la sexualidad, como se explicitó en los estudios de De Jesús (2018), Nieves (2012) y Ortiz (2016). Para esto no solo basta ser experto en la sexualidad sino también en política pública, política social y política educativa.

Se recomienda que sean tomado en cuenta los aspectos que se mencionan a continuación. Sería ideal (a) la realización de un estudio exhaustivo de las fallas en implementación habidas en las políticas públicas previas que han trabajado el tema de la educación sexual. Se encontró en las disertaciones doctorales revisadas 
que había desconocimiento de parte de los educadores en torno a las políticas públicas y lo que se requería de las mismas. Por lo cual este tipo de investigación daría paso a cerrar esas brechas, pues una vez se hallé el desfase, el mismo podría ser enmendado. Por otro lado, se destaca (b) que el enfoque que se le dé a la política pública educativa tenga su alcance con la niñez; que se tome en cuenta los cambios no solo generacionales sino también en las etapas de desarrollo. El currículo de educación sexual debe estar adaptado a cada etapa de desarrollo y tomando en cuenta la idiosincrasia la cultura en Puerto Rico. Entiéndase, se debe visibilizar el tabú que hay de la sexualidad (para erradicarlo) y la percepción de los padres y educadores en torno a cómo se deben educar los niños a nivel de sexualidad. Por último, se propone (c) visibilizar a los niños como sujetos de su propio derecho; tal como lo expusieran Freire (1997) y Smith (2000), ese derecho del educado a participar activamente del currículo. $Y$ tal como se recomendó en el estudio de Mazuera-Arias et al. (2018), esa acción en los estudiantes a participar en la política los empodera, los hace participes y responsables de sus actos, lo cual les permite esa valoración personal (p. 182). Entonces, se constituiría el ver a los estudiantes como actores de sus derechos.

A tono con lo anterior, se debe retomar lo explicitado en el Currículo de Salud en torno a la "integración de las madres, padres y encargados [...] con el fin de promover la colaboración entre el hogar y la escuela" (Compendio de Políticas del Departamento de Educación, Serie 410, p. 106). Los padres sí deben ser integrados y sí debe ser tomado su sentir en torno a lo que ellos piensan y siente sobre la sexualidad; pero, el Estado tiene también que tomar parte. Como se explicó antes, es una responsabilidad compartida. Tener la colaboración de los padres fortalecería lo que se presente en el currículo sexual, puesa ellosles tocaría fomentar la sexualidad saludable desde el hogar. Se toma en cuenta lo expuesto por Mazuera-Arias et al. (2018) en torno a que "la educación sexual debe ser considerada como una dimensión complementaria de la formación integral de las personas, que requiere la comunicación y la orientación adecuada de los padres" (p. 177). El que el niño tenga ese conocimiento y ese apoyo a nivel no solo escolar sino familiar, le daría la ventaja de poner de manifiesto sus derechos y tener en cuenta que el Estado no los abandonaría en caso de ser cometido algún tipo de abuso en su contra. Se sostiene que el Estado debe tener una participación educativa activa y liberadora de 
todo prejuicio. Tomando en cuenta lo hallado el artículo publicado por Martínez-Ramos (2013) en torno a la preparación de los educadores quienes darían los talleres a los docentes en el tema de educación sexual y lo expuesto en Currículo de Salud (Compendio de Políticas del Departamento de Educación, Serie 410), se recomienda que sean personas expertas en el tema quienes tengan a su cargo ese currículo de educación sexual en el sistema público del País.

A la luz de lo antes expuesto, está la brecha establecida debido a que se desconoce a qué responderán los educadores, si a lo expuesto en la Ley 85 del 2018, en torno la decisión de los padres o lo indicado en la serie 410 del Currículo de Educación Sexual. Se recomienda hacer investigaciones rigurosas para contestar las siguientes preguntas: "¿quéeraloquerealmentese deseaba implementar con la Ley 85 del 2018?”, "¿entenderían los progenitores qué era lo que el Gobierno deseaba para los niños y su derecho a recibir educación sobre la sexualidad?", "¿qué pasaría con los padres que practican el fundamentalismo religioso (interpretación literal de los textos religiosos)?", "si los padres no han recibido educación formal sobre la sexualidad, ¿qué es lo van a transmitir a sus hijos? ¿Cómo es lo que van a hacer?". Finalmente, se deja establecido la necesidad de la revisión de esta partícula del artículo 11.01, Derechos de los padres, tutores y encargados en torno a que estos pueden "seleccionar, de forma exclusiva, la forma y manera que se educarán sus hijos respecto a la sexualidad y afectividad" ( $p$. 79) para que pueda ser vinculada con el Compendio de Políticas del Departamento de Educación (en el cual está establecido el Currículo de Salud Sexual) y que esto vaya cimentado al mejor bienestar de los niños, las niñas y los/as jóvenes de Puerto Rico. 


\section{Referencias}

Carta Circular Núm. 2-2011-2012.

(2011). Política pública de educación en salud sexual en las escuelas públicas del

Departamento de Educación.

http://intraedu.dde.pr/Cartas\%20

Circulares/02-2011-2012.pdf

Carta Circular Núm. 3-2004-2005. (2004). Política pública de educación en salud sexual en las escuelas públicas del Departamento de Educación.

Carta Circular Núm. 19-20142015. (2015). Política pública sobre la equidad de género y su integración al currículo del Departamento de Educación de Puerto Rico como instrumento para promover la dignidad del ser humano y la igualdad de todos y todas ante la ley. Departamento de Educación. Estado Libre Asociado de Puerto Rico. http://intraedu.dde.pr/Cartas\%20 Circulares/19-2014-2015.pdf

Carta Circular Núm. 32-20162017. (2017). Política pública sobre la equidad de género y su integración al currículo del Departamento de Educación de Puerto Rico como instrumento para promover la dignidad del ser humano y la igualdad de todos y todas ante la ley. Departamento de Educación.
Estado Libre Asociado de Puerto Rico. http://intraedu.dde.pr/Cartas\%20 Circulares/32-2016-2017.pdf

Carta de los Derechos del Niño, Ley Núm. 338 del 31 de diciembre 1998. Leyes de Puerto Rico. http://www.lexjuris.com/lexlex/ley1998/ lex98338.htm

Compendio de Políticas del

Departamento de Educación, Serie 410. http://www.de.gobierno.pr/ files/compendio/SERIE A 400.pdf

Cruz-García, D., \& López-Opina, A. (2019). El Trabajo Social, democracia y política pública. Voces desde el Trabajo Social, 7 (1), 86-109. https://doi.org/10.31919/ voces.v7i1.76

De Jesús-Rosa, I. (2018). La atención del abuso sexual infantojuvenil en Puerto Rico desde la Ley 158 del 2013: Un estudio cualitativo a partir del paradigma de los derechos humano (Disertación doctoral inédita). Universidad de Puerto Rico, San Juan, PR.

De Jesús-Rosa, I, \& Jusino-Sierra, F. (2017). El trabajo social forense en la atención del abuso sexual infanto-juvenil en Puerto Rico: Un análisis crítico desde la perspectiva de los derechos humanos. En S. Amaro \& C. S. Krmpotic (Eds.). Diccionario 
internacional de trabajo social en el ámbito socio-jurídico (pp. 519542). Nova Casa Editorial.

Dewey, John. (1978). Democracia y educación: Una introducción a la filosofía de la educación (9na Ed.) [traducido al español de Lorenzo Luzuriaga]. Buenos Editorial Losada S.A.

Eisikovits, Z., Tener, D., \& LevWiesel, R. (2017). Adult women survivors of intrafamilial child sexual abuse and their current relationship with the abuser. American Journal of Orthopsychiatry, 87(3), 216-225. https://doi.org/10.1037/ort0000185

Fontes, L., \& Plummer, C. (2010). Cultural issues in disclosures of child sexual abuse. Journal of Child Sexual Abuse, 19(5), 491518. https://doi.org/10.1080/10538712.20 10.512520

Freire, P. (1997). Pedagogía de la autonomía: Saberes necesarios para la práctica educativa. Siglo XXI Editores.

Gardiner, M., \& Tenuto, P. (2016). Exploring ethical decisionmaking: Preparing organizations for the next century. Journal of Adult Education, 6-8.
Gonçalves Salgado, R., \& De Souza, L. (2018). Gêneros, sexualidades e infâncias: Cenas de crianças na contramão da inocência. Childhood \& Philosophy, 14(29), 241-258. https://biblioteca.uprrp. edu:2086/10.12957/childphilo.2018.30540

Kenny, M., \& Wurtele, S. (2013). Child sexual behavior inventory: A comparison between latino and normative samples of preschoolers. Journal of Sex Research, 50(5), 449-457. https:// doi.org/10.1080/00224499.2011.652265

Ley de Reforma Educativa de Puerto Rico, Ley Núm. 85 del 29 de marzo de 2018. Leyes de Puerto Rico.http://www2.pr.gov/ogp/BVirtual/ LeyesOrganicas/pdf/85-2018.pdf

Ley para la Seguridad, Bienestar y Protección de Menores, Ley Núm. 246 del 16 de diciembre de 2011. Leyes de Puerto Rico. http://www.lexiuris.com/lexlex/Leyes2011/ lex|2011246.htm

Littleton, F. (2014). How teen girls think about fertility and the reproductive lifespan: Possible implications for curriculum reform and public health policy. Human Fertility, 17(3), 180-187. https://doi.org/10.3109/14647273.2014.942389 
López-Bobonis, R., \& Rivero-Vergne, A. (2015). La victimización secundaria en las familias durante un proceso de alegación de abuso sexual infantil. En A. Rivero, R. Berríos, \& I. Romero (Eds). La aportación de la investigación cualitativa al campo de la salud en Puerto Rico.

Publicaciones Puertorriqueñas.

Machado das Neves, A., \& Ribeiro da Silva, I. (2018). Aspectos motivacionales de los maestros en proyectos de igualdad de derechos a las personas LGBTT en la escuela. Revista de Psicología y Ciencias Afines, 15(1), 7-14.

Martínez-Ramos, L. (2013).

Una historia de las políticas educativas relacionadas con género en Puerto Rico. Pedagogía, 46(1), 93-109.

Mazuera-Arias, R., Albornoz-Arias, N., Vivas-García, M., CarreñoParedes, M., Cuberos de Quintero, M., Hernández Lalinde, J., \& Bermúdez, V. (2018). Influencia de la educación sexual en la maternidad adolescente en el Estado Táchira, Venezuela. Archivos Venezolanos de Farmacología y Terapéutica, 37(3), 176-183.
Miragoli, S., Camisasca, E., \& Di Blasio, P. (2017). Child sexual behaviors in school context: Age and gender differences. Journal of Child Sexual Abuse, 26(2), 213231. https://doi.org/10.1080/10538712.20

\subsection{6}

Montero-Ossandón, L., DoisCastellón, A., DomínguezHidalgo, C., Valverde-Forttes, P., \& Bicocca-Gino, M. (2017). La educación sexual: Un desafío para la educación católica. Educación y Educadores, 20(3), 343-363. doi:10.5294/ edu.2017.20.3.1

Morales-Alejandro, L. (2018). Influencia de la religión en las prácticas sexuales de las personas adolescentes en Puerto Rico. Voces desde el Trabajo Social, 6(1), 18-43. https:// doi.org/10.31919/voces.v6i1.120

Nieves-Rolón, I. (2010). La política pública de educación en salud sexual en Puerto Rico: Implementación, proceso educativo y visión sobre los géneros. Cuaderno de Investigación en la Educación, 25, 137-154. http://cie.uprrp.edu/cuaderno/ wp-content/uploads/sites/4/2016/03/ cuaderno vol25 07.pdf 
Nieves-Rolón, I. (2012). El varón adolescente y su educación sexual: Consonancias y disonancias entre la política pública de educación en salud sexual en las escuelas públicas del Departamento de Educación y la socialización sexual de los varones adolescentes (Disertación doctoral inédita). Universidad de Puerto Rico, San Juan, PR.

Ortiz-Resto, V. (2016). Implantación de la política pública de educación en salud sexual en varias escuelas de la zona rural de Puerto Rico (Disertación doctoral inédita). Universidad de Puerto Rico, San Juan, PR.

Pulido Chaves, O. (2017). Política pública y política educativa: Una reflexión sobre el contexto. Revista Educación y Ciudad, (33), 13-28. https://doi.org/10.36737/01230425. v0.n33.2017.1641

Rondina Scandola, E. (2017).

Migración, refugio y trata de personas: Los (no)derechos en las políticas sociales. En S. Amaro, \& C. S. Krmpotic (Eds.). Diccionario internacional de Trabajo Social en el ámbito sociojurídico (pp. 519-542). Nova Casa Editorial.
Rorty, R. (1982). Consequences of pragmatism. University of Minnesota.

Smith, R. (2000). Whose childhood? The politics of homework. Children \& Society, 14(4), 316-325.

Steinhart, K., von Kaenel, A., Cerruti, S., Chequer, P., Gomes, R., Herlt, C., \& Horstick, O. (2013). International networking for sexuality education: A politically sensitive subject. Sex Education, 13(6), 630-643. https://doi.org/10.1080/1 4681811.2013 .784194

Tener, D. (2018). The secret of intrafamilial child sexual abuse: Who keeps it and how? Journal of Child Sexual Abuse, 27(1), 1-21. doi: 10.1080/10538712.2017.1390715

Torres, R. (2012, 27 de enero). Sociedad y educación: Perspectivas teóricas y metodológicas. http:// ingredientesinactivos.blogspot.com/2012/01/ sociedad-y-educacionperspectivas.html

UNESCO/Organización de las Naciones Unidas para la Educacion, la Ciencia y la Cultura. (2014). Educación integral de la sexualidad: Conceptos, enfoques y competencias. https://unesdoc.unesco.org/ark:/48223/ pf0000232800 
Venegas, M. (2013). Sex and relationships education and gender equality: Recent experiences from Andalusia (Spain). Sex Education, 13(5), 573-584. https://doi.org/10.1080/146818

\subsection{3 .778823}

Vygotsky, L.. (1997). Educational psychology [traducido al español de Robert Silverman]. St. Lucie Press.

Wilson, E., Dalberth, B., Koo, H., \& Gard, J. (2010). Parents' perspectives on talking to preteenage children about sex. Perspectives on Sexual \& Reproductive Health, 42(1), 56-63.

https://doi.org/10.1363/4205610 